\title{
Influencia en los signos vitales tras la administración de anestésico local con vasoconstrictor
}

Vital signs variations after local anesthetic with vasoconstrictor administration

\author{
Influência nas sinais vitais após a administração de anestésico local com \\ vasoconstritor
}

\author{
Daniela A. Obando-Moreno \\ daom_90@hotmail.com \\ Kleber A. Vallejo-Rosero" \\ avallejo@uce.edu.ec
}

Recibido: 9 de noviembre de 2016 * Corregido: 19 de diciembre de $2016 *$ Aceptado: 3 de enero 2017

\begin{abstract}
'Odontóloga, Facultad de Odontología, Universidad Central del Ecuador, Quito, Ecuador.
IIDocente, Facultad de Odontología, Universidad Central del Ecuador, Quito, Ecuador.
\end{abstract}




\section{Resumen.}

Objetivo: Determinar la influencia en los signos vitales luego de la administración de anestésico local con vasoconstrictor (lidocaína al 2\% con epinefrina 1:80.000). Materiales y métodos: El presente estudio fue de campo, comparativa y longitudinal, se realizó en 40 pacientes sanos de entre 21 a 24 años de edad, ambos sexos, sin antecedentes de enfermedades sistémicas preexistentes. Los pacientes fueron monitorisados con un monitor de signos vitales multiparámetro (OMNI Express) y sometidos a tres mediciones de signos vitales, antes, durante y después de 15 minutos de la administración de anestésico local con vasoconstrictor, usando la técnica de anestesia local toncular mandibular método directo, los resultados fueron pocesados por el paquete estadistico SPSS version 20, se aplico los test estadistico de la t student. Resultados: Se demostro que hay influencia del anestésico local con vasoconstrictor sobre los signos vitales en el $91 \%$ de los pacientes sin ser una alteración de significación clínica. El pulso se elevó con un promedio de 11 latidos por minuto entre la primera y segunda medicion. La presión arterial sistólica aumento 4,5 $\mathrm{mmHg}$ mientras que la diastólica aumento 2,5 $\mathrm{mmHg}$ ente la primera y segunda medicion respectivamente. La temperatura se elevó con un promedio de $0,39^{\circ} \mathrm{C}$ entre la primera y segunda medicion. Las respiraciones disminuyeron en un promedio de 3,9 entre la primera y segunda toma, y la saturación se elevó en un promedio de 0,9\% entre la primera y segunda toma. Conclusiones: Estadística y clínicamente de demostró la influencia en los signos vitales durante y después de la administración de anestésico local con vasoconstrictor (Lidocaína al 2\% con Epinefrina 1:80.000).

Palabras clave: Signos vitales; anestésico; vasoconstrictor. 


\section{Abstract.}

Objective: Determine the influence on vital signs of local anesthetic with vasoconstrictor $(2 \%$ lidocaine with epinephrine 1: 80,000). Materials and methods: The present study was field-based, comparative and longitudinal; performed in 40 healthy patients aged 21 to 24 years, both genders, with no history of pre-existing systemic diseases. Patients were monitored with a multiparameter vital signs monitor (OMNI Express) and underwent three vital signs measurements: before, during and 15 minutes after administration of local anesthesia with vasoconstrictor, using mandibular block technique. Data analysis was made through statistical package SPSS version 20 student t statistical test. Results: $91 \%$ of patients showed variations in vital signs, without an alteration of clinical significance. Heart rate increased 11 beats per minute between the first and second measurements. Systolic blood pressure increased $4.5 \mathrm{mmHg}$ while diastolic blood pressure increased $2.5 \mathrm{mmHg}$ between the first and second measurements. Body temperature increased in a $0.39^{\circ} \mathrm{C}$ average between the first and second measurements. Breathing rate decreased by an average of 3.9 between the first and second measurements and oxygen saturation rate increased by an average of $0.9 \%$ between the first and second measurements. Conclusions: Variations on vital signs during and after administration of local anesthetic with vasoconstrictor (2\% Lidocaine with Epinephrine 1: 80,000) have been demonstrated clinically and statistically.

Key Words: Vital signs; anesthesia; vasoconstrictor. 


\section{Resumo.}

Objetivo: Determinar a influência sobre as sinais vitais após a administração do anestésico local com vasoconstritor (Lidocaína 2\% com Epinefrina 1:80000). Materiais e Métodos: O presente estudo foi de campo, comparativo e longitudinal, realizou-se em 40 pacientes saudáveis de entre 21 e 24 anos de idade, ambos sexos, sem antecedentes de doenças sistêmicas preexistentes. Os pacientes foram monitorados com um monitor de sinais vitais multiparâmetro (OMNI Express) e submetidos a três medições de sinais vitais, antes, durante e depois de 15 minutos de administrado o anestésico local com vasoconstritor, utilizando a técnica de anestesia local troncular mandibular método direto, os resultados foram processados pelo pacote estatístico SPSS versão 20, o teste $\mathrm{t}$ Student foi aplicado. Resultados: Demostrou-se que existiu influência do anestésico local com vasoconstritor sobre as sinais vitais em $91 \%$ dos pacientes, sem ser uma alteração de significância clínica. O pulso elevou-se numa media de 11 batimentos por minuto entre a primeira e segunda medição. A pressão arterial sistólica aumentou 4,5 $\mathrm{mmHg}$ e a diastólica aumentou 2,5mmHg entre a primeira e segunda medição respetivamente. A temperatura elevou-se numa media de 0,9\% entre a primeira e segunda medição. As respirações por minuto diminuíram numa media de 3,9 entre a primeira e segunda toma. Conclusões: Estatística e clinicamente demostrou-se a influência nas sinais vitais durante e após a administração do anestésico local com vasoconstritor (Lidocaína 2\% com Epinefrina 1:80000).

Palavras chave: Sinais vitais; anestésico; vasoconstritor. 


\section{Introduccion.}

El anestésico local es uno de los grandes avances de la ciencia siendo en la actualidad un fármaco de uso cotidiano, utilizado para controlar el dolor durante los procedimientos clínicos y quirúrgicos. (1) Los anestèsicos son fàrmacos relativamente seguros con varias ventajas como la inhibición de la transmisión del impulso nervioso, bienestar para el paciente y el profesional en el momento de ejecutar los diversos procedimientos clínicos quirúrgicos, disminución de la toxicidad y tener un campo operatorio seco durante una cirugía. (2)

Sin embargo también son sustancias químicas que pueden provocar efectos de toxicidad que generalmente aparecen tras la inyección intravascular accidental o debido a una sobredosis, manifestándose principalmente en el sistema nervioso central y en el aparato cardiovascular. (3) La administración del anestésico local en dosis inadecuadas puede producir efectos adversos como: alteración de la presión arterial, alteración en la frecuencia cardíaca, alteración en la respiración, y la temperatura, es decir altera la función o fisiología normal de estos aparatos y sistemas. (1)

En Norteamerica y Europa e incluso en Latinoamérica, se han realizado diferentes estudios sobre los efectos que se produce en el organismo por la administración inadecuada de los anestésicos locales, (2) pero en nuestro país no existe antecedentes de ningún estudio con respecto a este tema. De ahí que el interés en realizar esta investigación es para tratar de entender el manejo farmacológico de los anestésicos locales y prevenir estas complicaciones.

Bajo estos circunstancias, se pretendió demostrar en el presente estudio, que el uso de lidocaína asociada a un vasoconstrictor provoca cambios a nivel de los signos vitales tales como la presión arterial, la frecuencia cardíaca y la respiración, signos propios de toxicidad; pudiendo llegar a constituir un factor de riesgo para el paciente si se coloca estas soluciones de manera inadecuada. 


\section{Materiales y métodos.}

Se realizó un estudio observacional, longitudinal. La muestra estuvo conformada por 40 pacientes voluntarios con un rango de edad de 21 a 24 años, de ambos sexos, sin antecedentes de enfermedades sistémicas preexistentes. El estudio se realizó en la clínica de Cirugía de la FOUCE para lo cual primero se hizó la valoración médica de los paciente, luego firmaron el consentimiento informado; despues de un reposo de 15 minutos se procedió a la monitorización con el monitor de signos vitales multiparámetro (OMNI Express), se realizó el registro de los signos vitales antes, inmediatamente después y después de 15 minutos de la administración del anestésico local mediante la técnica de anestesia troncular mandibular método directo para los nervios dentario inferior, lingual y bucal largo. Se realizó una comprobación objetiva del efecto anestésico con un elemento romo en lugares padronizados, luego una comprobación subjetiva del efecto anestésico en la región anatómica correspondiente a los nervios dentario inferior, lingual y bucal largo. Realizados los tres registros se evaluó la variación de los signos vitales.

Para la recolección de datos se elaboró un cuadro que consta con los siguientes datos: nombre del paciente, edad, sexo, ficha clínica, pulso, presión arterial, temperatura, respiración y oxigenación para las tres tomas respectivamente, se realizó una base de datos digital mediante el pograma SPSS 20 lo que nos permitió elaborar tablas estadísticas y sus respectivos gráficos para obtener los resultados, se utilizó la prueba de t student para comparar las medias de dos grupos diferentes. Es una prueba paramétrica para variables numéricas de distribución normal. La prueba t student, arroja el valor del estadístico t. Según sea el valor de t, corresponderá un valor de significación estadística determinado. Por medio de la prueba Kolmogorov - Smirnov Se demostró distribución normal. 


\section{Resultados.}

El registro del pulso inmediatamente después de la administración del anestésico presentó un aumento de 11 pulsaciones por minuto respecto del pulso previo a la administración del anestésico. El pulso a los 15 minutos registra una disminución de 5.57 pulsaciones por minuto respecto del pulso inicial. (Grafico $\left.\boldsymbol{N}^{\circ} \mathbf{1}\right)$

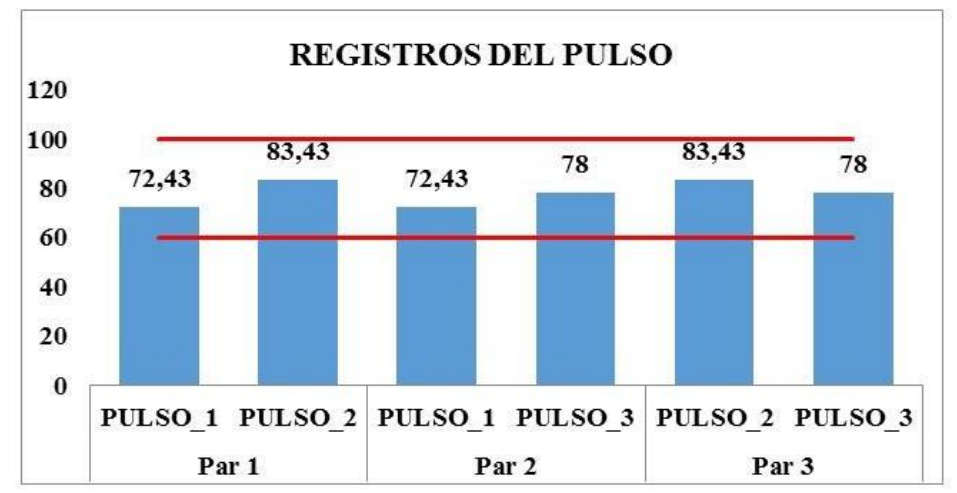

\section{Grafico $N^{\circ} 1 .-$ Comparación de los registros del pulso}

La presión sistólica inmediatamente después de la administración del anestésico registró un aumento de 4,25 mmHg respecto de la presión inicial. La presión sistólica después de 15 minutos de la administración del anestésico registró una disminución de $7.6 \mathrm{mmHg}$ respecto de la presión inicial. (Grafico $\left.\mathrm{N}^{\circ} 2\right)$

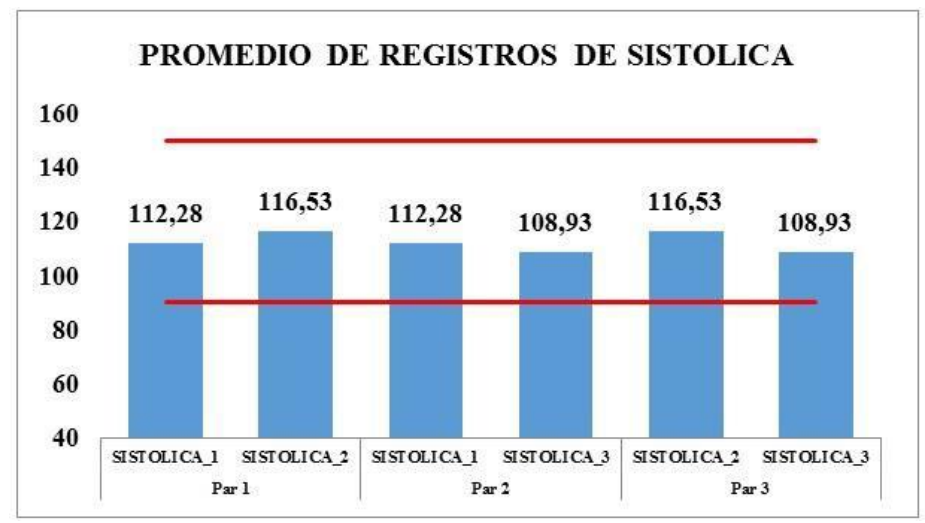

Grafico $N^{\circ}$ 2.- Comparación de los registros de la presión sistólica 
La presión diastólica inmediatamente después de la aplicación del anestésico registró un aumento de $2.3 \mathrm{mmHg}$ respecto de la presión diastólica previa a la administración del anestésico. La presión diastólica a los 15 minutos de la aplicación del anestésico registró una disminución de 6.1 $\mathrm{mmHg}$ respecto de la presión diastólica inicial.

La temperatura inmediatamente después de la administración del anestésico registró un aumento de $0.39{ }^{\circ} \mathrm{C}$ respecto de la temperatura previo a la administración del anestésico. La temperatura a los 15 minutos registra un nuevo aumento de $0.31^{\circ} \mathrm{C} \mathrm{mmHg}$ respecto a la temperatura inicial. (Grafico $\left.N^{\circ} 3\right)$

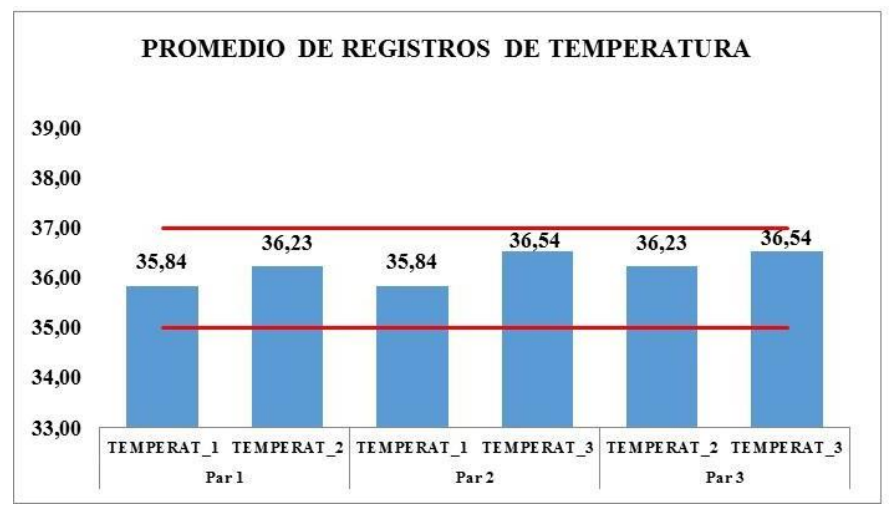

\section{Grafico $N^{\circ}$ 3.- Comparacion de los registros de la temperatura}

La respiración inmediatamente después de la administración del anestésico registró una disminución de 3.87 respiraciones por minuto respecto de la respiración previa a la administraciòn del amestèsico. La respiración 15 minutos después de la administración del anestésico registra un aumento de 2,1 respiraciones por minuto respecto de la respiración inicial. (Gráfico $N^{\circ} 4$ ) 


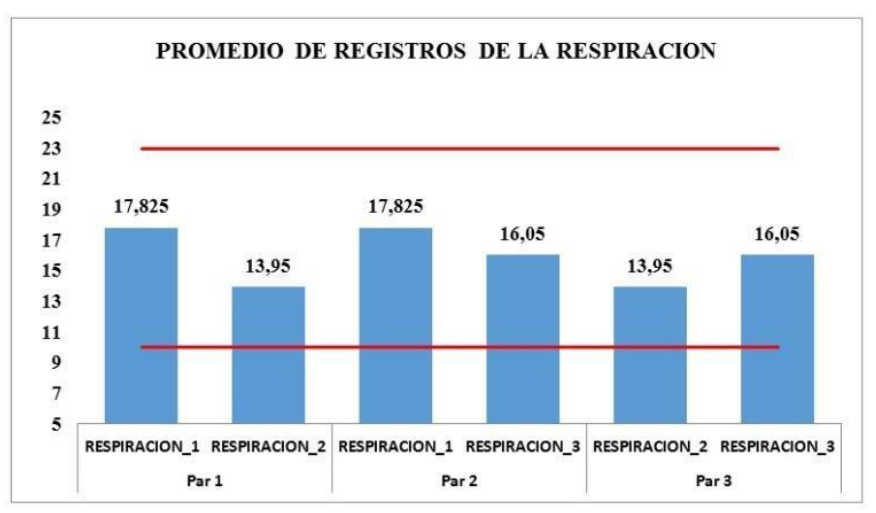

\section{Grafico $\mathrm{N}^{\circ}$ 4.- Comparacion de los registros de la respiración}

En el registro de la saturación 1comparada con la saturación 2 hay un aumento de $0.9 \%$. En la comparación de la saturación 2 vs la saturación 3 esta registra un descenso 1.8\%. (Gráfico $\left.\boldsymbol{N}^{\circ} \mathbf{5}\right)$

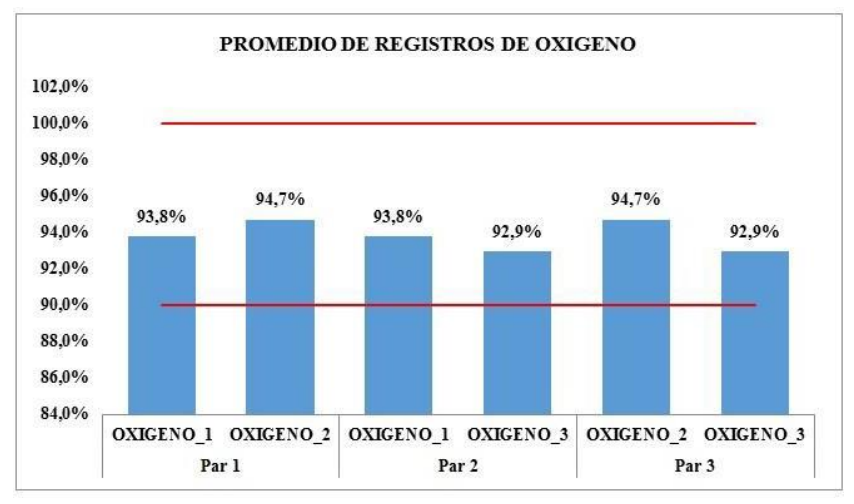

\section{Grafico $N^{\circ}$ 5.- Comparacion de los registros de la saturación}

\section{Discusión.}

El pulso aumenta luego de la inyección del anestésico, disminuyendo a sus valores normales luego de un período de 30 minutos, (4) registro que concuerda con los resultados de esta investigación donde el pulso tuvo cambios significativos.

Buffil observó que el pulso estuvo alto desde la administración del fármaco y 15 minutos después aumentó en la mayoría de los pacientes, lo que no coincidió con esta investigación. (5) 
Los pacientes adultos que fueron sometidos a anestesia local con vasoconstrictor no mostraron incrementos en la tensión arterial, $(6,5)$ lo cual no concuerda con esta investigación pues sí se presentó una variación de los valores de la presión arterial en un $20 \%$ en la primera toma y un $50 \%$ en la segunda toma.

Varios autores también manifestaron un incremento en la presión arterial, $(4,7,8)$ datos que se asemejan a los resultados de este estudio. Replogue K; et al, 2000, (9) valoraron la frecuencia cardíaca después de infiltrar Lidocaína más Epinefrina y apreciaron un ligero aumento en la presión arterial y pulso cardíaco.

Ryhänen JM; et al, 2001, (10) controló la presión sistólica y diastólica además del pulso y observó mínimos efectos en estos signos vitales. Según Conrado VC; et al, 2007, (11) los cambios detectados en su estudio son independientes de la presencia del vasoconstrictor y la dosis, asociándolos a factores ambientales e individuales de cada paciente

Niwa H; et al, 2001, (12)concluyen que no existen consecuencias hemodinámicas en los pacientes, lo que coincide con este estudio.

López J, (4) mencionaron respecto a la saturación de oxígeno en los pacientes estudiados, una variación entre $97.9 \%$ y $98.3 \%$, dato que concuerda con este estudio donde se observó una diferenciación de 93,8\% y 94.7\%. en la segunda toma, Benerjee N; 2006, (13) a nivel de la saturación, registraron un aumento transitorio pero que no fue clínicamente significativo, en esta investigación el aumento del porcentaje de saturación también fue transitorio.

En relación a la temperatura, de toda la bibliografía revisada no se encontraron estudios que involucre análisis de su influencia por la administración de lidocaína con vasoconstrictor. 


\section{Conclusiones.}

Luego de la administración de Lidocaína al 2\% con Epinefrina 1:80.000, los signos vitales de los pacientes, se vieron influenciados, con tendencia a un aumento en los mismos.

Se demostró que existe cambios a nivel del pulso cardíaco tras la administración de anestésico local con vasoconstrictor (Lidocaína al 2\% con Epinefrina 1:80.000).

Al analizar la presión arterial, se pudo constatar variaciones con tendencia a aumentar, tras la administración de anestésico local con vasoconstrictor (Lidocaína al 2\% con Epinefrina 1:80.000).

Se determinó que la administración de anestésico local con vasoconstrictor (Lidocaína al 2\% con Epinefrina 1:80.000) produjo variaciones en los valores de la temperatura con tendencia a aumentar.

La administración de anestésico local con vasoconstrictor (Lidocaína al 2\% con Epinefrina 1:80.000) influyó en los valores del número de respiraciones por minuto con tendencia a disminuir.

La saturación sanguínea demostró cambios con tendencia a aumentar la administración de anestésico local con vasoconstrictor (Lidocaína al 2\% con Epinefrina 1:80.000). 


\section{Bibliografia.}

1. Malamed-Stanley F. Manual de Anestesia Local. 6th ed. Madrid: Elsevier; 2014.

2. García-Iglesias B, Diéguez-García P, López-Álvarez S, Salamanca-Montaña E, Cobian-Llamas J. Toxicidad sistémica por anestésicos locales tras bloqueos de nervios periféricos. Rev. Esp. Anestesiol. Reanim. 2006; 53(8): p. 505-508.

3. Velázquez L. Farmacología Básica y Clínica. 18th ed. Madrid: Panamericana; 2008.

4. Lópe-Marcos J, García-Valle S, García-Cenador B. Anestésicos locales en odontología: valoración mediante pulsioximetría. Acta odontol. venez. 2006 ene; 44(1).

5. Buffil C. Efectos Adversos de la Anestesia Local en Odontología Buenos Aires: Comunicaciones Científicas y Tecnológicas; 2005.

6. Aboites-Morales A, Linares-Segovia B, Covarrubias-Rodríguez D, Núñez-Lemus E. Efecto de la Lidocaína con epinefrina en la tensión arterial de una población infantil. Rev Med Inst Mex Seguro Soc. 2008; 46(3): p. 323-327.

7. Neves R, Neves I, Giorgi D, Grupi C, César L, Hueb W, et al. Effects of epinephrine in local dental anesthesia in patients with coronary artery disease. Arq Bras Cardiol. 2007 may; 88(5): p. 545-551.

8. Mohammad K, Sadighi-Shamami M, Maryam A, Sadighi-Shamami M. Influence of local anesthetics with or without epinephrine $1 / 80000$ on blood pressure and heart rate: A randomized double-blind experimental clinical trial. Dent Res J (Isfahan). 2012 agos; 9(4): p. 437-440.

9. Replogle K, Reader A, Nist R, Beck M, Weaver J, Meyers W. Cardiovascular effects of intraosseous injections of 2 percent lidocaine with 1:100,000 epinephrine and 3 percent mepivacaine. J Am Dent Assoc. 1999 may; 130(5): p. 649-657.

10. Ryhänen J, Kotilainen R, Luotio K, Mattila M. Lidocaine and prilocaine with vasoconstrictors as cause for cardiovascular reactions. A pulse oximetric study. Preliminary report. Oral Surg Oral Diagn. 1996; 7(1): p. 21-24.

11. Conrado V, De-Andrade J, De-Angelis G, De-Andrade A, Timerman L, Andrade M, et al. Cardiovascular effects of local anesthesia with vasoconstrictor during dental extraction in coronary patients. Arq Bras Cardiol. 2007 may; 88(5): p. 507-513.

12. Niwa H, Sugimura M, Satoh Y, Tanimoto A. Cardiovascular response to epinephrine-containing local anesthesia in patients with cardiovascular disease. Oral Surg Oral Med Oral Pathol Oral Radiol Endod. $2001 \mathrm{dec}$; 92(6): p. 610-616.

13. Banerjee N. Tensión Arterial: etiología y tratamiento. 2nd ed. India: Jain Publishers; 2006. 